\title{
Composition chimique, dégradabilité in sacco et produits de fermentation in vitro de la fétuque élevée (Festuca arundinacea)
}

\author{
ZOFFOUN G. Alex ${ }^{1,2 *}$, ABOH B. André1,2, MBANZAMIHIGO Léonidas ${ }^{3}$ et FIEVEZ Veerle ${ }^{3}$ \\ 1'Laboratoire de Recherches Zootechnique, Vétérinaire et Halieutique (LRZVH), Institut National des Recherches \\ Agricoles du Bénin (INRAB), 01 BP 884 Recette Principale, Cotonou, République du Bénin (West Africa). 2Laboratoire \\ d'Ecologie Appliquée, Faculté des sciences Agronomiques, Université d'Abomey-Calavi 01 BP 526 Cotonou, Bénin. \\ 3 Université de Gand, Département de Production Animale, Proefhoevestraat 10, 9090 Melle (Belgique). \\ *Corresponding author. Tel.: (+229) 96697153; E-mail address: zofalex@yahoo.fr, alex.zoffoun35@gmail.com.
}

Original submitted in on 30th June 2013 Published online at www.m.elewa.org on 30th July 2013.

https://dx.doi.org/10.4314/jab.v67i0.95014

\section{RÉSUMÉ}

Objectifs : En vue d'une sélection pour une utilisation comme aliment pour bétail, vingt quatre écotypes de fétuque élevée (Festuca arundinacea) provenant du milieu naturel du nord de la Belgique ont été étudiés afin d'en déterminer la valeur nutritionnelle.

Méthodologie et Résultats : La composition chimique, la dégradabilité in sacco et les incubations in vitro ont été réalisés. Un mouton adulte fistulé au niveau du rumen a été utilisé pour la détermination de la dégradabilité in sacco des écotypes et les incubations in vitro. La teneur moyenne en MAT (8,0\% MS) des 24 écotypes étudiés est faible. Les écotypes de Festuca arundinacea présentent en moyenne une meilleure dégradabilité potentielle $(a+b)(82,7 \%)$ que les fourrages tropicaux Pennisetum purpureum $(64,9 \%)$ et Tripsacum laxum $(66,8 \%)$ mais moindre que les fourrages tempérés (Lolium perenne: 90,5\%). Festuca arundinacea a présenté une production élevée de propionate $(234.7 \mu \mathrm{mol} / \mathrm{mmol}$ AGV totaux).

Conclusion: Les meilleurs de la collection de 24 écotypes de F. arundinacea ont été identifiés pour une utilisation comme aliment pour bétail. Les teneurs en MAT trouvées pour la fétuque élevée sont comparables à celles des fourrages tropicaux qui sont généralement plus pauvres en protéine que les fourrages tempérés ; ce qui représente un handicap pour leur ingestion volontaire. Ces résultats impliquent d'une part qu'il faut une supplémentation en azote pour les pâturages de Festuca arundinacea et d'autre part l'exploitation des pâturages à la fétuque élevée au stade avant frutification. Par ailleurs, la culture en association de fétuque élevée et légumineuses est également une solution envisageable. Une variabilité a été notée entre les écotypes de Festuca arundinacea qui en moyenne présentent une meilleure dégradabilité que les fourrages tropicaux, mais moindres que les fourrages tempérés. Cet avantage de la fétuque élevée sur les fourrages tropicaux se consolide par une production élevée d'acide propionique et faible d'acide acétique comparables à celles des fourrages tempérés ; ce qui est très intéressant car le propionate peut générer du glucose, un des facteurs limitant pour la production laitière.

Mots clés: Festuca arundinacea, écotype, azote ammoniacal, dégradabilité in sacco, méthane, acides gras volatiles 
Chemical composition, in sacco degradability and in vitro fermentation patterns of tall fescue (Festuca arundinacea)

\section{Abstract}

Objectives: In order to select for use as livestock feed, twenty four ecotypes of tall fescue (Festuca arundinacea) obtained from the natural environment in northern Belgium were studied to determine the nutritional value.

Methodology and Results: Chemical composition, in sacco degradability and in vitro incubations were performed. One rumen fistulated adult sheep was used for the determination of in sacco degradability and in vitro incubations study. The mean CP content ( $8.0 \%$ of DM) of all the 24 ecotypes was lower. $F$. arundinacea ecotypes have on average a better potential degradability $(a+b)(82.7 \%)$ than that of tropical grasses Pennisetum purpureum (64.9\%) and Tripsacum laxum (66.8\%), but less than that of temperate grasses (Lolium perenne: $90.5 \%)$. F. arundinacea showed a high propionate production $(234.7 \mu \mathrm{mol} / \mathrm{mmol}$ total VFA).

Conclusion: The best of the collection from 24 ecotypes $F$. arundinacea have been identified for use as cattle feed. The CP contents found for tall fescue are comparable to tropical forages which are generally lower in protein than temperate forage, representing a handicap for their voluntary ingestion. These results imply firstly the need for supplemental nitrogen to $F$. arundinacea pastures and secondly the use of tall fescue pastures before frutification. Moreover, the association pasture of tall fescue and legumes is also an option. Some variability was observed between ecotypes of $F$. arundinacea that show on average better degradability than tropical forages, but less than the temperate forage. This advantage of tall fescue on tropical forages is consolidated by an high production of propionic acid and low acetic acid comparable to temperate forage, this is very interesting since propionate can generate glucose, one of the limiting factors for dairy production.

Key-words: Festuca arundinacea, ecotype, ammonia nitrogen, in sacco degradability, methane volatile fatty acids.

\section{INTRODUCTION}

Le réchauffement progressif de la planète terre devient de plus en plus une réalité. Selon le troisième rapport du Groupe Intergouvernemental d'Experts sur le Climat (GIEC), l'augmentation des températures de surface moyennes globales sera de 1 à $3^{\circ} \mathrm{C}$ vers 2100 (GIEC, 2001). Ces modifications inquiétantes du climat auront des répercussions néfastes sur l'équilibre de l'écosystème et donc sur les productions végétales en général et les pâturages en particulier (GIEC, 2007). Dans ces conditions, des espèces fourragères adaptées aux températures élevées, telle que la fétuque élevée (Festuca arundinacea), devront être sélectionnées pour une utilisation à grande échelle. Festuca arundinacea est une graminée perenne cespiteuse, parfois rhizomateuse (rhizomes courts) et à port érigé pouvant atteindre jusqu'à $2 \mathrm{~m}$ de haut (Terrell, 1979). La fétuque élevée est endémique à plusieurs pays de l'Europe et de l'Afrique du Nord (Borril et al., 1971). II est difficile de définir de manière précise son origine. Des études réalisées par Borrill (1972) ainsi que Hunt et Sleper (1981) ont permis d'identifier l'Atlas Marocain comme l'origine probable de Festuca arundinacea. C'est l'un des fourrages les plus utilisés aux Etats Unis par exemple (Hopkins and Alison, 2006 ; Hoveland, 2009 ; Samples et al., 2009). Plusieurs études ont montré que la fétuque élevée est une graminée fourragère qui présente de multiples qualités agronomiques telles que la pérennité, l'adaptation à des conditions difficiles et la productivité élevée. Elle s'adapte aussi bien aux régions chaudes, froides, humides que sèches (Horst et Nelson, 1979 ; King et Bush, 1985 ; Carrow et Duncan, 2003; Hoveland, 2009). Malgré ces mutiples qualités, les défauts majeurs de la fétuque élevée sont sa médiocre appétibilité et sa faible digestibilité (Emile et al., 1992; Kallenbach et al., 2003 ; Flores et al., 2008). Peu d'études ont été réalisées en Belgique sur la fétuque élevée bien que cette plante existe dans les pâturages naturels. La présente étude a pour objectif d'évaluer la valeur alimentaire d'une collection de 24 écotypes de F. 
arundinacea des pâturages naturels du nord de la Belgique en vue d'un choix pour une meilleure

\section{MATÉRIELS ET MÉTHODES}

Matériel végétal et analyses chimiques : Vingt quatre écotypes de $F$. arundinacea ont été collectés dans les pâturages naturels du nord de la Belgique et replantés, sur le site expérimental du laboratoire de Production Animale de Gand à Melle, sur un sol pauvre en azote. Quarante six jours après, les écotypes replantés ont été coupés à une hauteur de $5 \mathrm{~cm}$ au dessus du sol, puis soumis à un séchage doux $\left(45^{\circ} \mathrm{C}, 72 \mathrm{~h}\right)$. Après séchage, une partie de chaque échantillon a été moulue sous tamis de $1 \mathrm{~mm}$ pour analyser son contenu en matière sèche (MS), matière organique (MO), matières minérales (MM), matières azotées totales (MAT), sucres solubles dans l'eau (SS), Neutral Detergent Fibre (NDF) et Acid Detergent Fibre (ADF) (AOAC, 1990). alimentation du bétail.

Animal et ration alimentaire : Un bélier castré de race Texel mixte porteur d'une fistule permanente au niveau du rumen et pesant $97 \mathrm{~kg}$ a été utilisé pour les expérimentations in sacco et in vitro. L'animal est gardé dans une cage métabolique et recevait du foin (Lolium perenne) ad libitum deux fois par jour à $9.00 \mathrm{~h}$ et à 16.00 $\mathrm{h}$ avec seulement $2 \times 100 \mathrm{~g}$ de concentré commercial pour simuler une ration de base riche en fibre. La consommation journalière moyenne de foin était de $962 \pm$ $145 \mathrm{~g}(\mathrm{n}=27)$ de MS. Des échantillons ont été prélevés sur tous les sacs de concentré et sur toutes les bottes de foin pour en déterminer la composition chimique. La composition chimique du foin et du concentré sont rapportés dans le tableau 1.

Tableau 1: Composition chimique de la ration du mouton d'expérimentation

\begin{tabular}{lcccccccc}
\hline \hline & \multicolumn{7}{c}{ Composition chimique } \\
\cline { 2 - 8 } & MS & MO & MM & CellB & NDF & ADF & MGB & MAT \\
\cline { 2 - 8 } & 84,5 & 91,4 & 8,6 & - & 56,8 & 25,4 & - & 9,9 \\
\hline Foin & & & & & $\%$ MS & & & \\
Concentré & 90,2 & 88,9 & 11,1 & 8,9 & - & - & 4,4 & 18,3 \\
\hline \hline
\end{tabular}

MS : matière sèche ; MO : matière organique ; MM : Matières minérales ; CellB : cellulose brute ; NDF : Neutral Detergent Fibre ; ADF : Acid Detergent Fibre ; MGB : graisses brutes ; MAT : matières azotées totales ; - : non déterminé.

Afin de caractériser le milieu ruminal du mouton d'expérimentation au cours de l'évaluation de la dégradabilité in sacco, le pH et la concentration en azote ammoniacal $\left(\mathrm{N}^{-} \mathrm{NH}_{3}\right)$ dans le jus du rumen ont été mésurés comme suit : Juste avant le repas du matin ( 0 h), 1, 3 et $7 \mathrm{~h}$ après. L'azote ammoniacal a été dosé par la méthode de microdiffusion de Conway (Conway, 1957).

Dégradabilité in sacco : Les mêmes échantillons de $F$. arundinacea moulus sous tamis de $2 \mathrm{~mm}$, ont servi pour l'étude de la dégradabilité in sacco. Pour chaque échantillon de $F$. arundinacea, 6 sachets en polyamide (longueur $14 \mathrm{~cm}$; largeur $5 \mathrm{~cm}$; mailles 37 micron) avec $2 \mathrm{~g}$ d'échantillon chacun ont été introduits dans le rumen du mouton puis retirés après $3,6,9,24,72$ et 96 heures. Le septième sachet (non introduit dans le rumen) sert à estimer la fraction soluble. Les sachets retirés du rumen, ainsi que celui non introduit étaient lavés $2 \times 10$ minutes à la machine puis séchés à $65^{\circ} \mathrm{C}$ pendant 48 heures et pesés. Après déduction du pourcentage de matière sèche (MS) dégradée dans le rumen, ces résultats ont été traités suivant l'équation $P=a+b\left(1-e^{-c t}\right)(\varnothing r s k o v$ et McDonald, 1979) où : $P=$ dégradabilité au temps $t(\%) ; a$ $=$ fraction soluble ou rapidement dégradable $(\%) ; b=$ fraction potentiellement dégradable $(\%) ; c=$ vitesse de dégradation de $b\left(h^{-1}\right) ; t=$ temps $(h)$.

Incubations in vitro: La dégradabilité in sacco permet de savoir la part de l'échantillon qui disparaît dans le rumen, mais ne donne pas d'information sur les produits de dégradation; d'où la nécessité de faire des incubations in vitro. Les paramètres étudiés lors des incubations in vitro sont: les productions d'AGV, de méthane et les bilans d'hydrogène métabolique. Les mêmes échantillons de $F$. arundinacea utilisés pour la dégradabilité in sacco ont servi comme substrat. Les incubations in vitro ont été réalisées avec du contenu du rumen prélevé avant la distribution du repas du matin puis filtré en anaérobiose sous un courant de $\mathrm{CO}_{2}$. Dix mililitres de jus de rumen mélangés avec $40 \mathrm{ml}$ d'une solution tampon de phosphate $(\mathrm{pH}=7)$ sont incubés au bain-marie mouvant $\left(39^{\circ} \mathrm{C} / 24 \mathrm{~h}\right)$ dans des flacons sous atmosphère de $\mathrm{CO}_{2}$ avec $500 \mathrm{mg}$ de chaque écotype de $F$. arundinacea. Trois 


\section{Zoffoun et al J. Appl. Biosci. 2013. Composition chimique, dégradabilité in sacco et produits de fermentation in vitro de la fétuque élevée (Festuca arundinacea)}

répétitions sont réalisées par écotype et un flacon incubé sans substrat servait de témoin. Après l'incubation, la fermentation était stoppée en injectant dans chaque flacon $1 \mathrm{ml}$ d'un mélange d'acide phosphorique et formique (10:1). La détermination de la production de $\mathrm{CH} 4$, se faisait en injectant $2 \mathrm{ml}$ de gaz total prélevé sur chaque flacon dans un chromatographe à gaz. Après détermination de la production de methane, les flacons sont ouverts et leur contenu homogénéisé, centrifugé, filtré et conservé au réfrigérateur pour les analyses d'acides gras volatiles (AGV) par chromatographie en phase gaseuse. La production nette de $\mathrm{CH}_{4}$ et d'AGV est calculée en soustrayant les productions dans le flacon témoin de celles des flacons incubés avec substrat. Les bilans d'hydrogène métabolique de chaque incubation ont été calculés à l'aide des équations stoechiométriques établies par Demeyer (1991) :

\section{RÉSULTATS}

Composition chimique des écotypes de $F$. arundinacea : Les différentes composantes chimiques des écotypes de $F$. arundinacea ont varié entre les valeurs suivantes en $\%$ de $\mathrm{MS}$ : $\mathrm{MO}(87,8$ à $92,6 \%)$, MM (7,4 à 12,2\%), NDF (49,0 à 61,7 \%), ADF (24,8 à 32,8 $\%)$, SS (17,0 à 19,7) et MAT (6,4 à 12,7\%). Les écotypes
Bilan $2 \mathrm{H}$ métabolique

$=(2 \mathrm{H}$ utilisé $/ 2 \mathrm{H}$ produit $) \times 100$

$=\left[\left(2 \mathrm{P}+2 \mathrm{~B}+4 \mathrm{CH}_{4}\right) /(2 \mathrm{~A}+\mathrm{P}+4 \mathrm{~B})\right] \times 100$

et où

$\mathrm{C}_{6}$ fermenté $\left(\mathrm{C}_{6} f\right)=\mathrm{A} / 2+\mathrm{P} / 2+\mathrm{B}$

Traitements des résultats : L'analyse statistique des résultats obtenus a été réalisée à l'aide du logitiel SPSS version 9.0. Pour mettre en évidence l'existence de différences significatives entre les écotypes pour les paramètres étudiés, la méthode d'analyse de variance a été utilisée, complétée par un test "Duncan" permettant de comparer les moyennes entre les groupes. Les correlations entre les différents paramètres ont été analysées avec le test "Pearson Correlations".

14 et 15 ont une plus faible teneur en NDF de 49,0 et $53,9 \%$ respectivement. L'écotype 15 a également le taux de MAT le plus élevé (12,7\%). Les teneurs en NDF et MAT des écotypes de $F$. arundinacea sont rapportés dans la figure 1.

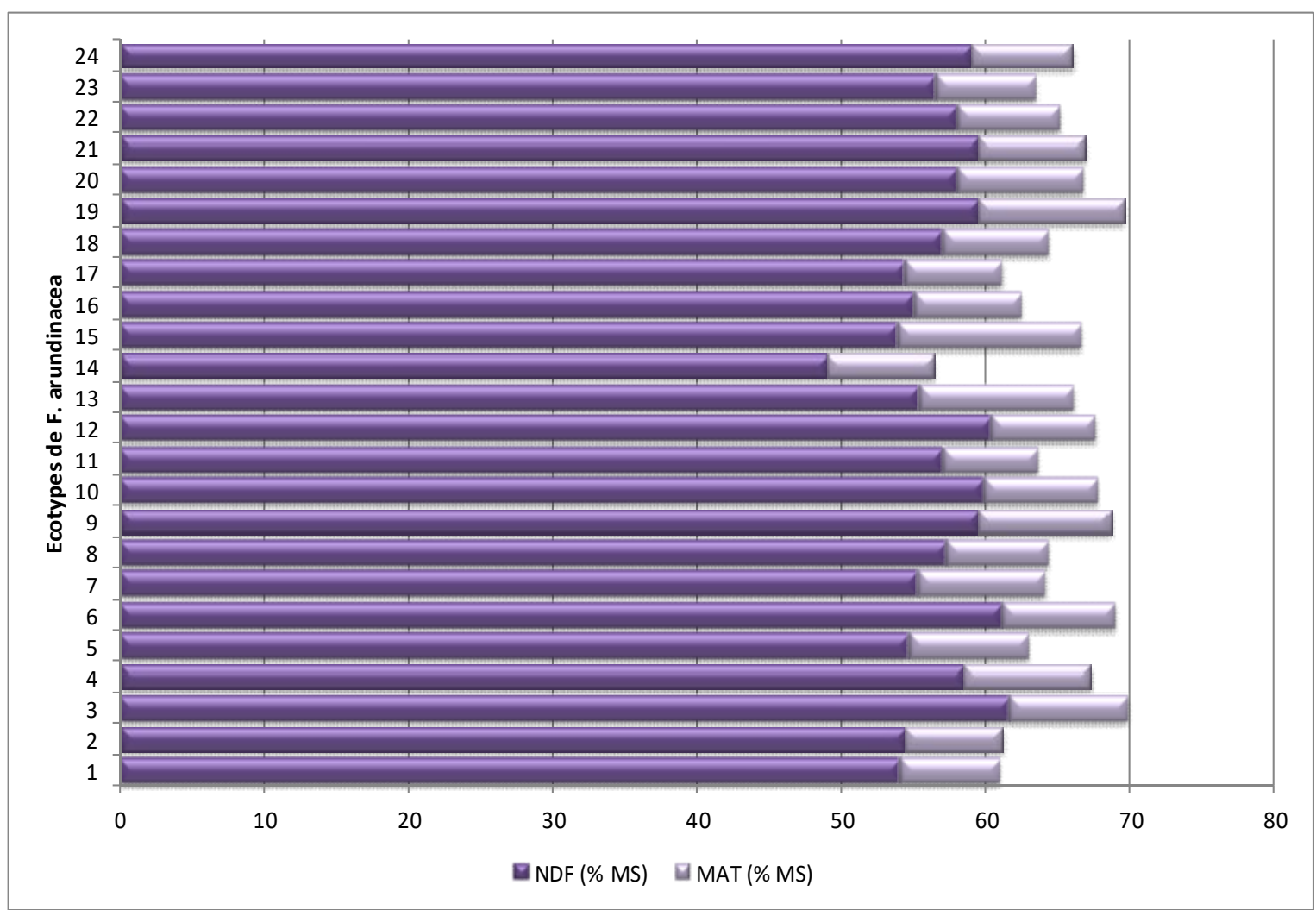

Figure 1: Teneur en NDF et MAT des 24 écotypes de $F$. arundinacea 
Dégradabilité in sacco des écotypes de $F$. arundinacea : Les valeurs du $\mathrm{pH}$ du contenu du rumen du mouton d'expérimentation ont oscillé entre 6,1 et 6,8 .
Une diminution $\mathrm{du} \mathrm{pH}$ a lieu une heure après la distribution de la nourriture du matin et remonte sensiblement sept heures après (figure 2).

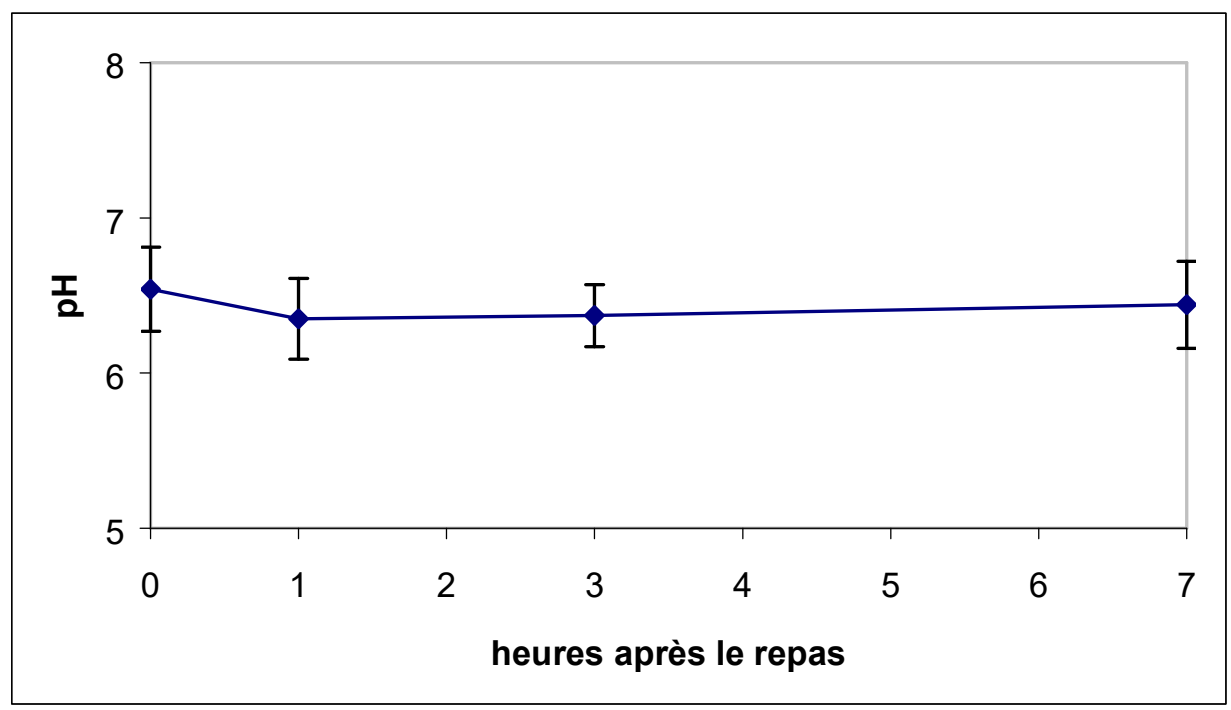

Figure 2 : Variations du pH du rumen entre les 2 distributions de nourriture

La concentration en azote ammoniacal du jus de rumen du mouton d'expérimentation a varié entre 51,1 et 119,0 $\mathrm{mg} /$ litre. Ce taux d'azote ammoniacal a évolué au cours de la journée, il augmente après la distribution de la nourriture du matin pour former un plateau entre une heure et trois heures avant de diminuer graduellement jusqu'à son niveau le plus bas sept heures après (figure 3).

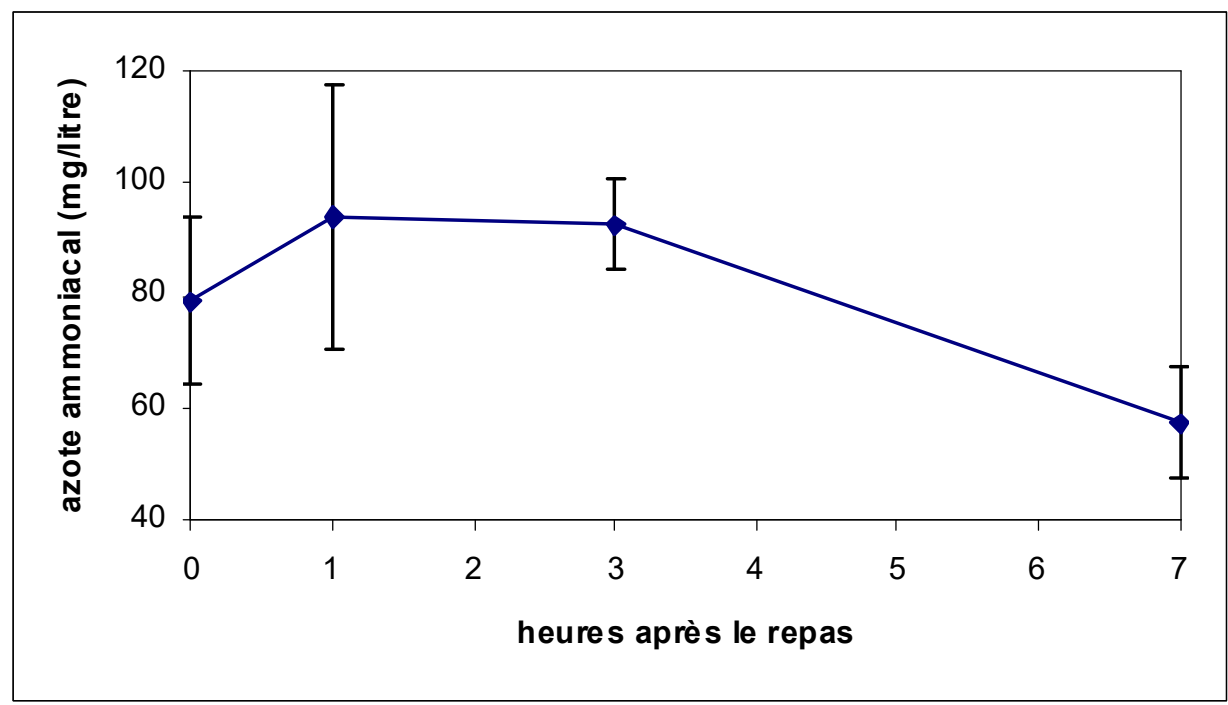

Figure 3 : Variations de la concentration en $\mathrm{N}-\mathrm{NH}_{3}$ du rumen entre les 2 distributions de nourriture

Pour les 24 écotypes de $F$. arundinacea étudiés, la dégradabilité potentielle $(a+b)$ a varié entre 72,1 et $89,5 \%$ de MS. La vitesse de dégradabilité de $b$ quant à elle est comprise entre 2,2 et $4,5 \% \mathrm{~h}^{-1}$. L'exemple de courbe de dégradation représentée à la figure 4 montre que le modèle de Ørskov et McDonald (1979) a été suivi. En prenant en compte les maxima pour les paramètres de dégradabilité in sacco, les écotypes 14 et 1 se distinguent des autres respectivement par une plus grande dégradabilité potentielle $(a+b)(89.5 \%$ versus 
$72.1 \%)$ et une plus grande vitesse de dégradation $\left(4,5 \% \quad h^{-1}\right.$ versus $\left.2,2 \% h^{-1}\right)$.

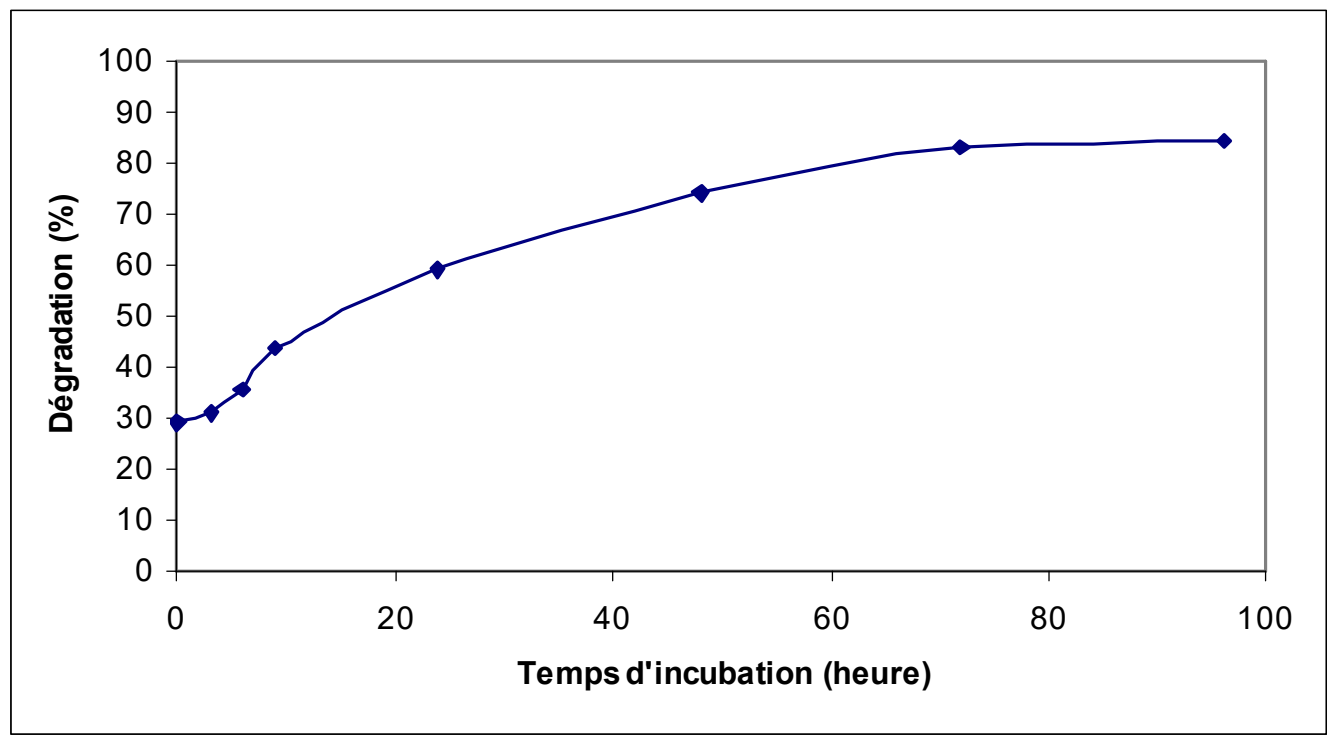

Figure 4 : Exemple de courbe de dégradation d'un écotype de F. arundinacea

Les produits de fermentation in vitro des écotypes de F. arundinacea : Les pourcentages des AGV totaux sont compris dans les intervalles suivants: acétate $(59,5$ à $68,2 \%)$, propionate $(19,8$ à $25,6 \%)$, butyrate $(11,1$ à $13,0 \%)$, méthane (21,7 à $28,2 \mu \mathrm{mol} / \mathrm{mmol}$ AGV totaux). Les bilans d'hydrogène quant à eux ont varié entre 93,3 et 77,0 avec une moyenne de $86,2 \%$. La figure 5 rapporte l'analyse de variances entre écotypes pour les productions d'AGV totaux. II se dégage de cette figure que les écotypes 14, 1 et 2 ont produit plus d'AGV totaux à l'opposé de l'écotype 18.

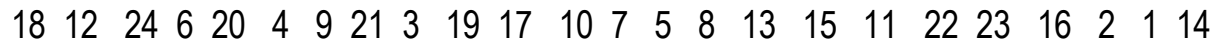

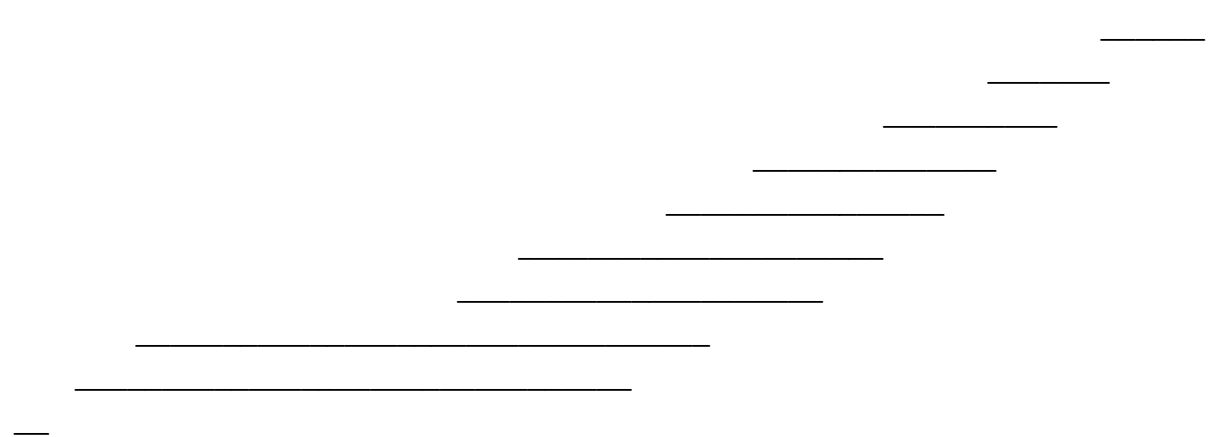

Les écotypes se retrouvant au dessus des lignes différentes sont significativement différents $(p<0.05)$.

Figure 5 : Production par ordre croissant d'AGV totaux des 24 écotypes de $F$. arundinacea

Correlations entre les paramètres étudiés: Les correlations entre les divers paramètres étudiés revèlent une correlation positive entre NDF et acétate, par contre les correlations NDF / dégradabilité, NDF / proportion de propionate et NDF / AGV totaux sont négatives. Les correlations NDF / vitesse de dégradabilité et NDF / production de $\mathrm{CH}_{4}$ ne sont pas significatives (tableau 2). 
Tableau 2 : Correlations entre les paramètres étudiés

\begin{tabular}{lrc}
\hline \hline Paramètres correlés & $\mathbf{r}$ & $\mathbf{p}$ \\
\hline NDF / a+b & $-0,454$ & 0,026 \\
NDF / c & $-0,219$ & 0,305 \\
NDF / Acétate & 0,437 & 0,033 \\
NDF / Propionate & $-0,450$ & 0,028 \\
NDF / Méthane & 0,341 & 0,103 \\
NDF / AGV totaux & $-0,690$ & 0,000 \\
\hline \hline
\end{tabular}

MS : matière sèche ; MO : matière organique ; NDF : Neutral Detergent Fibre ; ADF : Acid Detergent Fibre ; MAT : matières azotées totales $; a$ : fraction soluble $; b$ : fraction potentiellement dégradable $; a+b$ : dégradabilité potentielle $; c:$ vitessede dégradation de $b$; $r$ : coefficient de correlation ; $p$ : probabilité de $r$.

\section{DISCUSSION}

Composition chimique des écotypes de $F$. arundinacea : A l'exeption de la teneur en MAT, la compositon chimique des écotypes de $F$. arundinacea étudiés est comparable à celles obtenues antérieurement par certains auteurs (Wilman et al., 1996; Wilman et Moghaddam, 1998 ; Kallenbach et al., 2003 ; Flores et al., 2007). En ce qui concerne les teneurs en MAT, les valeurs obtenues dans cette étude sont nettement inférieures à celles rapportées par ces auteurs $(8,0 \%$ vs 15,3 - 22,1\%). L'âge avancé de l'herbe à la coupe (46 jours) et probablement le manque d'azote dans le sol sont à l'origine de cette faible teneur en MAT. En moyenne, les résultats obtenus pour les teneurs en MAT de $F$. arundinacea sont comparables à celles des fourrages tropicaux qui ont généralement une moindre teneur en protéines $(5,34-10,12 \% \mathrm{MS})$ (Buldgen et al., 2001; Toléba et al., 2001 ; Bindelle et al., 2007 ; Aboh et al., 2008 ; Ajayi et al., 2008 ; Babatoundé et al., 2008 ; Eniolorunda et al., 2008 ; Adjolohoun et al., 2013). Cette faible teneur des fourrrages tropicaux en protéines est en grande partie à la base de la faible production enregistrée. En effet, Minson (1981) puis Preston et Leng (1987) font observer que la vitesse de croissance et la production de lait des animaux élevés uniquement sur des pâturages tropicaux sont généralement inférieures à celles obtenus lorsque ces mêmes animaux sont élevés sur des pâturages tempérés. Plusieurs auteurs ont indiqué qu'il faut un minimum de teneur en MAT ( $8 \%$ de MS) pour assurer un fonctionnement adéquat aux microorganismes du rumen (Van Soest 1982 ; Song et Kennelly 1990 ; Coleman et al., 2003 ; Norman et al., 2007). Ces auteurs ont observé que l'appétit de l'animal diminue lorsque la teneur en MAT de l'aliment descend en dessous du seuil de 6-8\% MS, et attribuent cette diminution de l'ingestion volontaire et à une insuffisance de la croissance microbienne dans le rumen qui ne favorise pas une dégradation rapide des substrats.
Au stade physiologique de 46 jours d'âge, seuls 9 écotypes de $F$. arundinacea sur les 24 étudiés possèdent un taux de MAT suffisant (8\%) pour favoriser une bonne ingestion et une meilleure croissance microbienne. II s'agit des écotypes 3, 4, 5, 7, 9, 13, 15, 19 et 20. L'utilisation des 15 écotypes restants nécessiterait donc une source d'azote complémentaire. Flachowsky et al. (1999) et Eniolorunda et al. (2008) rapportent que l'ingestion volontaire des fourrages est en relation avec leur teneur en fibres, mais les caractéristiques physiques de ces fibres sont également importantes. Ceci est confirmé par Mtengett et al. (1996) et Bindelle et al. (2007) qui ont observé que les différences morphologiques entre les fourrages font varier leur ingestion volontaire quand bien même leurs compositions chimiques sont similaires.

Dégradabilité in sacco des écotypes de $F$. arundinacea : Le pH du jus du rumen a varié entre 6,1 et 6,8. Cette variation du pH est dans les limites permettant une bonne cellulolyse. D'autres expériences avaient conclu que les valeurs du $\mathrm{pH}$ dans le rumen varient dans une fourchette relativement large de 5,5 à 7,0 (Dirksen, 1976; Fonty et al., 1995), mais des valeurs comprises dans une zone plus étroite entre 6,0 et 7,0 sont nécessaires pour une bonne activité microbienne (Jarrige, 1988; Russel, 1998 ; Flores et al., 2007). L'ammoniac est un précurseur important de la protéosynthèse microbienne. Plusieurs études menées aussi bien in vitro qu'in vivo ont montré qu'en général, les concentrations de $\mathrm{NH}_{3}$ dans le rumen comprises entre 30 et $80 \mathrm{mg} \mathrm{N} / \mathrm{l}$ suffisent à assurer la protéosynthèse microbienne maximale (Satter et Slyter, 1974; Durand, 1982 ; Song et Kennelly, 1990). Preston et Leng (1987) de même que Flores et al. (2008) ont également rapportées des valeurs plus élevées allant de 168 à 238 mg de $\mathrm{N}-\mathrm{NH}_{3} /$ litre de jus de rumen comme pouvant assurer une croissance microbienne maximale. Les concentrations en azote ammoniacal du jus de rumen de 
l'animal d'expérimentation étaient donc suffisantes $(51,1$ et $119,0 \mathrm{mg}$ / litre) pour favoriser une bonne croissance microbienne et une bonne dégradation des substrats incubés. En moyenne, les écotypes de F. arundinacea présentent une meilleure dégradabilité potentielle $(a+b)$ $(82,7 \%)$ que les fourrages tropicaux Pennisetum purpureum $(64,9 \%$ ) et Tripsacum laxum $(66,8 \%$ ) (Misson, 1990 ; Teguia et al., 1999). II existe une variabilité entre les écotypes de $F$. arundinacea quant à leur dégradabilité. Cette différence entre les écotypes est le résultat d'une différence entre leurs teneurs en fibres (Norman et al., 2007). La correlation positive entre la teneur en NDF et la dégradabilité des écotypes de $F$. arundinacea confirme cette idée. $F$. arundinacea présente une plus faible dégradabilité potentielle $(a+b)$ in sacco $(82,7 \%)$ que Lolium perenne, une graminée fourragère des pays tempérés (90,5\%) (Beever et Siddons, 1984 ; Bonos et al., 2004).

Les produits de fermentation in vitro des écotypes de $F$. arundinacea : Malgré la teneur élevée en fibres, $F$. arundinacea a produit une forte proportion de propionate (234,7 $\mu \mathrm{mol} / \mathrm{mmol}$ AGV totaux) comparable au mélange de ray grass et trèfle $(244,3 \mu \mathrm{mol} / \mathrm{mmol}$ AGV totaux) (Mbanzamihigo et al., 2000). Cette production importante de propionate est en accord avec le pourcentage élevé de sucres solubles $(18,2 \%)$ observé dans les écotypes de $F$. arundinacea étudiés. Ceci est d'une importance capitale quand on sait que le propionate peut générer du

\section{RÉFÉRENCES BIBLIOGRAPHIQUES}

Aboh AB, Ehouinsou MA, Olaafa M, Brun A, 2008. Complémentation alimentaire des ovins Djallonké avec les sous-produits de transformation d'ananas : potentiel nutritif, préférence et développement pondéral. Bull. Rech. Agron. Bénin 61: 25-30.

Adjolohoun S, Dahouda M, Adandedjan C, Houinato M, Toléba Séibou S, Sinsin B. 2013. Differences in biomass production and nutritive value of 9 Panicum maximum ecotypes in Soudanian region of West Africa. African Journal of Agricultural Research 8: 1661-1668.

Ajayi TF, Babayemi JO, Taiwo AA, 2008. Effects of supplementation of Panicum maximum with four herbaceous forage legumes on performance, nutrient digestibility and nitrogen balance in West African dwarf goats. Anim. Sci. J. 79: 673679.

Annicchiarico P. and Romani M, 2005. Genetic variation, heritability and genetic correlations for forage glucose par la néoglucogénèse chez l'animal. Le glucose est très important pour la synthèse du lactose du lait et pour la reproduction (Preston and Leng, 1987; Annicchiarico and Romani, 2005). Les proportions des différents AGV produits par $F$. arundinacea sont similaires à celle des fourrages tempérés qui produisent bien moins d'acétate $(60,2 \%)$ et plus de propionate $(23,3 \%)$ dans le rumen que les fourrages tropicaux. Les fourrages tropicaux quant à eux produisent une grande proportion d'acétate $(72,8 \%)$ et une faible proportion de propionate $(17,8 \%)$ dans le rumen (Minson, 1981 ; Bindelle et al., 2007). Demeyer (1981), Annison et Bryden (1998), de même que Norman et al. (2007) ont observé que les proportions relatives des différents A.G.V. dans le rumen dépendent surtout de la nature de la ration et que les régimes à base de fourrages orientent les fermentations vers l'acétate. Les rations riches en concentrés quant à elles orientent les fermentations plutôt vers le propionate tandis que les sous produits de sucrerie (mélasse) induisent une fermentation de type butyrique relativement importante. La correlation négative $(r=-0,690 ; p=$ $0,000)$ entre la teneur en NDF des écotypes de $F$. arundinacea et leurs productions d'AGV totaux de même que la correlation positive $(r=0,437, p<0,05)$ entre la teneur en NDF et la production d'acétate confirment que les différences de production d'AGV entre les fourrages tempérés et tropicaux seraient dûes à la teneur élevée en fibres de ces derniers.

quality and yield traits of Mediterranean tall fescue germplasm. Plant Breed. 124: 99-101.

Annison EF. and Bryden WL, 1998. Perspectives on ruminant and metabolism. I. Metabolism in the rumen. Nutr. Res. Rev., 11: 173-198.

AOAC 1990. Official Methods of Analysis. 15th Edn. (Association of Official Analytical Chemists, INC, Va. USA).

Babatounde S, Toleba SS, Adandedjan CC, Dahouda M, Sidi $H$, Buldgen A, 2008. Comportement alimentaire et évolution pondérale des moutons Djallonké sur des pâturages de fourrages cultivés en mélange. Ann. Sci. Agron. 10: 31-49.

Beever DE. and Siddons RC, 1984. In: L.P. Milligan, W. L. Grovum, A. Dobson (Editors), Digestion and metabolism in the grazing ruminant. Proceedings. Sixth International Symposium on Ruminant Physiology, (Canada, 10th-14th 1984) pp. 479-497.

Bindelle J, llunga $Y$, Delacollette $M$, Muland Kayij $M$, Umba DiM'Balu J, Kindele E, Buldgen A, 2007. 
Voluntary intake, chemical composition and in vitro digestibility of fresh forages fed to Guinea pigs in periurban rearing systems of Kinshasa (Democratic Republic of Congo). Trop. Anim. Health Prod. 39: 419-426.

Bonos SA, Rush D, Hignight K, Meyer WA, 2004. Selection for deep root production in tall fescue and perennial ryegrass. Crop Sci. 44: 17701775.

Borrill M, Tyler B, Lloyd-Jones M, 1971. Studies in Festuca. I. A chromosome atlas of bovinae and scariosae. Cytologia, 36: 1-14.

Buldgen A, Michiels B, Adjolohoun S, Babatounde S, Adandedjan CC, 2001. Production and nutritive value of grasses cultivated in the coastal area of Benin. Trop. Grassl. 35: 43-47.

Carrow RN. and Duncan RR, 2003. Improving drought resistance and persistence in turf-type tall fescue. Crop Sci. 43:978-984.

Coleman SW, Hart SP, Sahlu T, 2003. Relationships among forage chemistry, rumination and retention time with intake and digestibility of hay by goats. Small Rum. Res. 50: 129-140.

Conway DJ, 1957. Microdiffusion analysis and volumetric error, $4^{\text {th }}$ ed. London: Crosby Lockwood.

Demeyer D, 1981. Rumen microbes and and digestion of plant cell walls. Agric. Environ., 6: 295-337.

Demeyer D, 1991. Quantitative aspects of microbial metabolism in the rumen and hindgut. In: J. P. Jouany (Editor), Rumen Microbial Metabolism and Ruminant Digestion. I.N.R.A. Editions, Paris, pp. 217-237.

Dirksen G, 1976. L'acidose du rumen. Le Point Vét., 18: 9.

Durand M, 1982. Orientation du métabolisme du rumen. Ann. Zootech., 31: 47-76.

Emile JC, Gillet M, Ghesquière M, Charrier X., 1992. Pâturage continu de fétuque élevée par des vaches laitières: amélioration de la production par l'utilisation d'une variété sélectionnée pour l'appétibilité. INRA Fourrages 130: 159-169.

Eniolorunda OO, Jinadu OA, Ogungbesan MA, Bawala TO, 2008. Effect of combined levels of Panicum maximum and Gliricidia sepium on nutrient digestibilities and utilization by west African dwarf goats fed cassava offal based concentrate. Res. J. Anim. Sci. 2: 149-153.

Flachowsky G, Kamra DN, Zadrazil F, 1999. Cereal straws as animal feed - Possibilities and limitations. J. Appl. Anim. Res., 16: 105-118.
Flores R, Coblentz WK, Ogden RK, Coffey KP, Looper ML, West CP, Rosenkrans CF, 2007. Effects of fescue type, sampling date, and strip-grazing on the nutritive value and in situ ruminal disappearance kinetics of DM and NDF for autumn-stockpiled tall fescue forages. J. Dairy Sci. 90: 2883-2896.

Flores R, Coblentz WK, Ogden RK, Coffey KP, Looper ML, West CP, Rosenkrans CF, 2008. Effects of fescue type and sampling date on the $\mathrm{N}$ disappearance kinetics of autumn-stockpiled tall fescue. J. Dairy Sci. 91: 1597-1606.

Fonty G, Jouany J-P, Forano E, Gouet Ph, 1995. L'écosystème microbien du réticulo-rumen. In : Nutrition des ruminants domestiques; ingestion et digestion (R. Jarrige, Y. Ruckebush, C. Demarquilly, M.-H. Farce and M. Journet, Editors). I.N.R.A. Editions, Paris, pp. 299-347.

GIEC, 2001. Bilan 2001 des changements climatiques : Les éléments scientifiques Résumé à l'intention des décideurs. Contribution du Groupe de travail I au troisième rapport d'évaluation du Groupe d'experts intergouvernemental sur l'évolution du climat.

GIEC, 2007. Résumé technique. In: changements climatiques 2007: Les éléments scientifiques. Contribution du groupe de travail I au quatrième Rapport d'évaluation du Groupe d'experts intergouvernemental sur les changements climatiques [Solomon, S., D. Qin, M. Manning, Z. Chen, M. Marquis, K.B. Averyt, M. Tignor and H.L. Miller (eds.)]. Cambridge University Press, Cambridge, Royaume-Uni et New York, NY, Etats-Unis d'Amérique.

Hopkins AA. and Alison MW, 2006. Stand persistence and animal performance for tall fescue endophyte combinations in the south central USA. Agron. J. 98: 1221-1226.

Horst GL. And Nelson CJ, 1979. Compensatory growth of tall fescue following drought. Agron. J., 71: 559563.

Hoveland CS 2009. Origin and history. p. 3-10. In H.A. Fribourg, D.B. Hannaway, and C.P. West (ed.) Tall fescue for the twenty-first century. Agron. Monogr. 53. ASA, CSSA, and SSSA, Madison, WI.

Hunt KL. and Sleper DA, 1981. Fertility of hybrids between two geographic races of tall fescue. Crop Sci., 21: 400-404. 
Jarrige $\mathrm{R}, 1988$. Ingestion et digestion des aliments. In: Alimentation des bovins ovins et caprins. I.N.R.A. Editions, Paris, pp. 29-56.

Kallenbach RL, Bishop-Hurley GJ, Massie MD, Rottinghaus GE, West CP, 2003. Herbage mass, nutritive value, and ergovaline concentration of stockpiled tall fescue. Crop Sci. 43:1001-1005.

King MJ. and Bush LP, 1985. Growth and water use of tall fescue as influenced by several soil drying cycles. Agron. J., 77: 1-4.

Mbanzamihigo L, da Costa Gomez C, Fievez V, Piattoni F, Carlier L, Demeyer D, 2000. Nitrogen fertilisation effects on sward and methane production: importance of season and animal effects. Proceedings. 25e Studiedag voor Nederlandstalige Voedingsonder- zoekers, (Wageningen, The Netherlands; 28 April 2000), pp 38-39.

Minson DJ, 1990. The chemical composition and nutritive value of tropical grasses. Pages 163-180 dans P. J. Skermann et F. Riveros, eds. Tropical grasses. Plant production and protection series N822. FAO, Rome, Italy.

Minson DJ, 1981. Nutritional differences between tropical and temperate pastures. In: Grazing animals ( $\mathrm{F}$. H. W. Morley, Editor). World Animal Science, B 1: pp 143-157.

Mtengett EJ, Wilman D, Moseley G, 1996. Differences between twelve forage species in physical breakdown when eaten. Journal of Agriculture Science, Cambridge, 126: 287-293.

Norman RC, Coblentz WK, Hubbell DS, Ogden RK, Coffey KP, Rhein RT, West CP, Rosenkrans CF, 2007. Effects of bale diameter, storage location, and sampling depth on the nutritive value, ruminal in situ disappearance kinetics of dry matter, and concentrations of ergovaline within endophyte-infected tall fescue hay packaged in large round bales. Crop Sci. 47: 1635-1646.

Ørskov ER. and McDonald I, 1979. The estimation of protein degradability in the rumen from incubation measurements weighed according to rate of passage. J. Agric. Sci., Camb., 92: 499503.

Preston TR. and Leng RA, 1987. Matching ruminant production systems with avalable resources in the tropics and sub-tropics. Penanbul books Armidale, Australia, pp 245.
Russel JB, 1998. The importance of $\mathrm{pH}$ in the regulation of ruminal acetate to propionate ratio and methane production in vitro. J. Dairy Sci., 81: 3222-3230.

Samples TJ, Sorochan JC, Brilman LA, Stier JC, 2009. Tall fescue as turf in the United States. p. 445482. In H.A. Fribourg, D.B. Hannaway, and C.P. West (ed.) Tall fescue for the twenty-first century. Agron. Monogr. 53. ASA, CSSA, and SSSA, Madison, WI.

Satter LD. and Slyter LL, 1974. Effect of ammonia concentration on rumen microbial protein production in vitro. Br. J. Nutr. 32: 199-208.

Seehovic J, Poisson C, Gillet M, 1985. Palatability and organoleptic characteristics of grasses. I Comparison between ryegrass and tall fescue. Agronomie, 5 (4): 347-354.

Song MK. and Kennelly JJ, 1990. Ruminal fermentation pattern, bacterial population and ruminal degradation of feed ingredients as influenced by ruminal ammonia concentration. J. Anim. Sci., 68: $1110-1120$

Teguia A, Ørskov ER, Kyle DJ, 1999. Anote on in situ degradability and in vitro gas production of some West African grass species and multipurpose legume tree leaves. J. Anim. Feed Sci., 8: 415424.

Terrell EE, 1979. Taxonomy, morphology and phylogeny. P. 31-39. In Buckner R. C. et Bush L. P. (ed.) Tall fescue. Agron. Monogr., 20. ASA, CSSA, and SSSA, Madison, WI.

Toleba SS, Babatounde S, Troungnin H, Chabi SLW, Adandedjan CC, 2001. Etude comparative de deux espèces fourragères (Panicum maximum local et Brachiaria ruziziensis) complementées par des graines de coton sur les performances pondérales des ovins djallonké. Ann. Sci. Agron. Bénin 2: 193-208.

Van Soest PJ, 1982. The whole animal: Intake. In: Nutritional Ecology of the ruminant (P. J. Van Soest, Editor) pp: 276-293.

Wilman D. and Moghaddam PR, 1998. In vitro digestibility and neutral detergent fibre and lignin contents of plant parts of nine forage species. $J$. Agric. Sci., Camb., 131: 51-58.

Wilman D, Gao Y, Altimini MAK, 1996. Difference between related grasses, times of year and plant parts in digestibility and chemical composition. J. Agric. Sci., Camb., 127: 311318. 